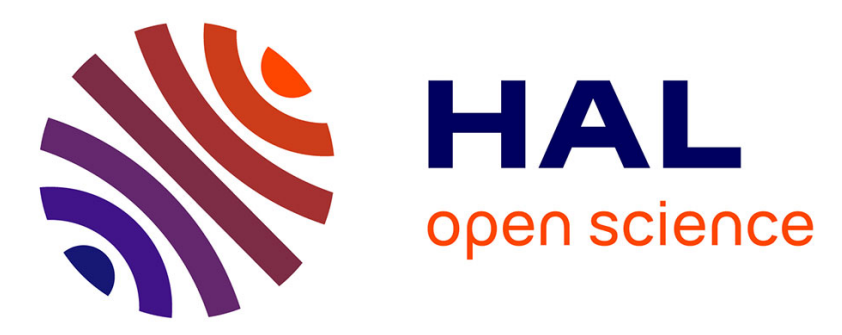

\title{
Synthesis of the Diastereomers of Dethiobiotin Using the Conjugate Addition of 4-Phenyloxazolidin-2-one to a Nitroalkene
}

Denis Lucet, Philippe Heyse, Arnaud Gissot, Thierry Le Gall, Charles Mioskowski

\section{To cite this version:}

Denis Lucet, Philippe Heyse, Arnaud Gissot, Thierry Le Gall, Charles Mioskowski. Synthesis of the Diastereomers of Dethiobiotin Using the Conjugate Addition of 4-Phenyloxazolidin-2-one to a Nitroalkene. European Journal of Organic Chemistry, 2000, 2000 (21), pp.3575-3579. 10.1002/10990690(200011)2000:213.0.CO;2-R . hal-02503542

\section{HAL Id: hal-02503542 \\ https://hal.science/hal-02503542}

Submitted on 10 Mar 2020

HAL is a multi-disciplinary open access archive for the deposit and dissemination of scientific research documents, whether they are published or not. The documents may come from teaching and research institutions in France or abroad, or from public or private research centers.
L'archive ouverte pluridisciplinaire HAL, est destinée au dépôt et à la diffusion de documents scientifiques de niveau recherche, publiés ou non, émanant des établissements d'enseignement et de recherche français ou étrangers, des laboratoires publics ou privés. 


\title{
Synthesis of the Diastereomers of Dethiobiotin Using the Conjugate Addition of 4-Phenyloxazolidin-2-one to a Nitroalkene
}

\author{
Denis Lucet, ${ }^{[a]}$ Philippe Heyse, ${ }^{[a]}$ Arnaud Gissot, ${ }^{[a]}$ Thierry Le Gall,*[a] and Charles \\ Mioskowski* ${ }^{*}[\mathbf{a}, \mathbf{b}]$
}

Keywords: Asymmetric synthesis / Conjugate addition / Dethiobiotin / Imidazolidin-2-one / Nitroalkene

[a] CEA-Saclay, Service des Molécules Marquées Bât. 547, 91191 Gif-sur-Yvette cedex, France Fax: (internat.) +33 (0)1 69087991 E-mail: legall@dsvidf.cea.fr

[b] Université Louis Pasteur, Laboratoire de Synthèse Bio-Organique associé au CNRS, Faculté de Pharmacie 74 route du Rhin, B. P. 24, 67401 Illkirch, France E-mail: mioskowski@ dsvidf.cea.fr

\begin{abstract}
Natural D-dethiobiotin and its three stereoisomers were pre-pared from a single nitroalkene 2. Conjugate addition of the potassium salt of $(R)$-4-phenyloxazolidin-2-one 3 to 2 led to two diastereomeric nitro compounds $\mathbf{6}$ and 7 . Their enantiomers were prepared from $(S)$-3. These compounds were converted in three analogous steps into the dethiobiotin isomers as their methyl esters.
\end{abstract}

In the course of a study into the biosynthesis of biotin, a vitamin which acts as a cofactor for carboxylase reactions, we were interested in the synthesis of the various diastereomers of the biotin precursor dethiobiotin 1. This cis-substituted imidazolidin-2-one, derived from the corresponding diamine, is converted into biotin under the action of the enzyme biotin synthase (Scheme 1). ${ }^{[1-3]}$

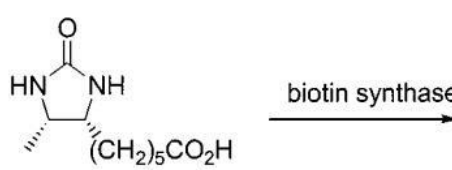

1

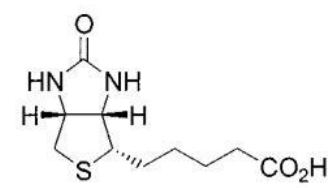

(+)-biotin

Scheme 1. Biosynthesis of biotin

In this article, we describe the synthesis of all four diastereomers of dethiobiotin. The retrosynthetic analysis for the dethiobiotin isomers is presented in Scheme 2. The two nitrogen-containing functional groups could be introduced using the conjugate addition of a nitrogen nucleophile to nitroalkene $\mathbf{2}$ of the appropriate chain length and the carboxylic function. We have previously reported the highly stereoselective conjugate addition of the potassium salt of 4-phenyloxazolidin-2-one 3 to monosubstituted nitroalkenes and the conversion of a corresponding adduct into an enantiomerically pure monosubstituted imidazolidin-2-one. ${ }^{[4,5]}$ When applied to disubstituted nitroalkenes, pairs of diastereomers should be obtained. Furthermore, since both enantiomers of $\mathbf{3}$ are commercially available, the method would allow for the synthesis of the four isomers of dethiobiotin.

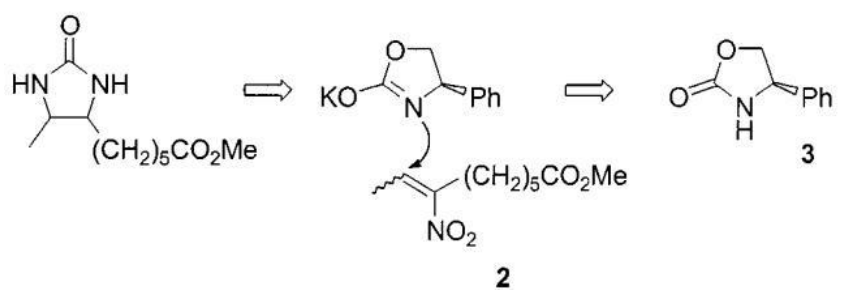

Scheme 2. Retrosynthetic analysis of dethiobiotin diastereomers

Nitroalkene 2 was prepared according to the sequence described in Scheme 3. Commercially available 7-bromoheptanenitrile was converted in three efficient steps into the known methyl 7-nitroheptanoate $4 .{ }^{[6]}$ Nitroaldolisation of $\mathbf{4}$ with acetaldehyde afforded nitro alcohol $\mathbf{5}$. Acetylation of $\mathbf{5}$ followed by treatment with basic alumina yielded nitroalkene 2 as a 90:10 mixture of $(E)$ - and $(Z)$-isomers. 


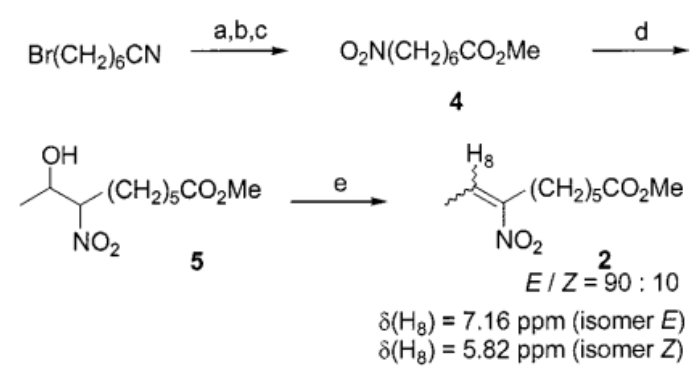

Scheme 3. Preparation of nitroalkene 2; reagents and conditions: (a) $\mathrm{H}_{2} \mathrm{SO}_{4}, \mathrm{MeOH}, 40 \mathrm{~h}$, reflux, 66\%; (b) NaI, acetone, 30h, reflux, 94\%; (c) $\mathrm{AgNO}_{2}$, ether, 3 days, room temp., 80\%; (d) $\mathrm{CH}_{3} \mathrm{CHO}, \mathrm{KOH}, \mathrm{MeOH}, 19 \mathrm{~h}, 0{ }^{\circ} \mathrm{C}, 84 \%$; (e) i) DMAP, $\mathrm{Ac} 2 \mathrm{O}$, ether, $16 \mathrm{~h}$, room temp., ii) DMAP, basic alumina, $4 \mathrm{~h}$, reflux, 63\% (two steps)

Conjugate additions of the potassium salt generated from either $(R)$ - or $(S)$-3, by treatment with potassium tert-butoxide in THF in the presence of 0.1 equivalent of 18-crown-6, with nitroalkene 2 were then performed. The results obtained from $(R)-3$ are summarized in Scheme 4 . After 45 minutes at $-78{ }^{\circ} \mathrm{C}$, the reaction mixture was quenched with acetic acid. Only two diastereomeric adducts were formed according to ${ }^{1} \mathrm{H}$ and ${ }^{13} \mathrm{C}$ NMR spectra of the crude product. Chromatography allowed for the separation of compounds 6 (anti-adduct) and 7 (syn-adduct). The indicated relative and absolute configurations were not known at this time, but derived by comparison of the final products with an authentic sample.

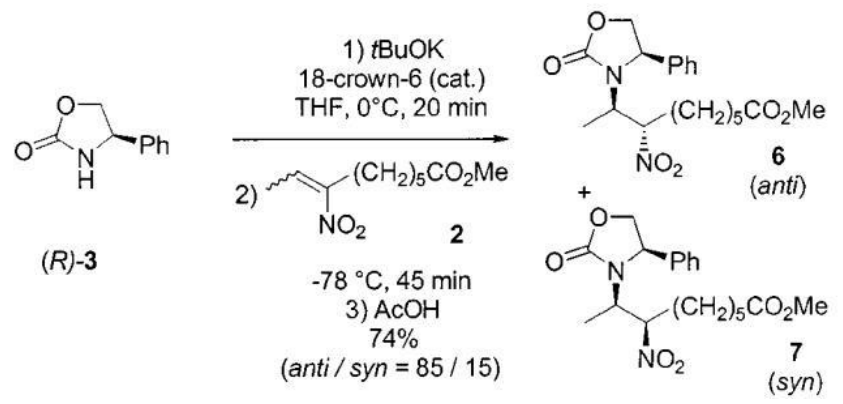

Scheme 4. Conjugate addition of the potassium salt of $(R)-\mathbf{3}$ on nitroalkene 2

The anti/syn ratio (85:15) was sufficient to carry out the following steps from the major adduct 6 . However, larger quantities of syn-adduct 7 were needed. For this purpose, the isomerization of compound $\mathbf{6}$ to its epimer 7 was briefly studied under various basic conditions, as described in Table 1. By stirring a solution of compound $\mathbf{6}$ in dichloromethane in the presence of triethylamine at room temperature, it was possible to obtain after 2.5 days a anti/syn ratio of 60:40, with no noticeable degradation. When this isomerization was applied to the crude product of the conjugate addition, a 46:54 anti/syn ratio was obtained, with an $83 \%$ combined yield of adducts.

Table 1. Study of the isomerization of anti-nitro compound 6

\begin{tabular}{|c|c|c|}
\hline Reagent & Conditions & 6/7 Ratio $^{[\mathrm{a}]}$ \\
\hline$\overline{\mathrm{MeONa}, \mathrm{MeOH}}$ & $3 \mathrm{~h}$ at reflux & {$[\mathrm{b}]$} \\
\hline $\begin{array}{l}\mathrm{K}_{2} \mathrm{CO}_{3}, \mathrm{THF} \\
\mathrm{K}_{2} \mathrm{CO}_{3}, \mathrm{MeOH}\end{array}$ & $\begin{array}{l}24 \text { h, room temp. } \\
24 \text { h, room temp. }\end{array}$ & 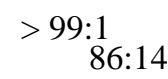 \\
\hline $\mathrm{Et}_{3} \mathrm{~N}, \mathrm{CH}_{2} \mathrm{Cl}_{2}$ & $60 \mathrm{~h}$, room temp. & $60: 40$ \\
\hline
\end{tabular}

The adducts 6 and 7, and their enantiomers ent-6 and ent-7 obtained from $(S)$-4-phenyloxazolidin-2-one were then all converted into the dethiobiotin methyl ester or into its stereoisomers in a similar manner as described in Scheme 5, starting from 6. Treatment of $\mathbf{6}$ with ammonium formate in the presence of palladium on carbon in methanol afforded the corresponding amine 8. Heating this compound at reflux with potassium hydroxide in methanol led to the more stable imidazolidinone 9. The methyl ester was hydrolyzed to a carboxylic acid under these conditions. The last step consisted of 
the hydrogenolysis of the remains of the original source of chirality, and the concomitant formation of the methyl ester, leading to imidazolidinone 10. The same sequence was carried out from nitro compound 7, as summarized in Scheme 6.

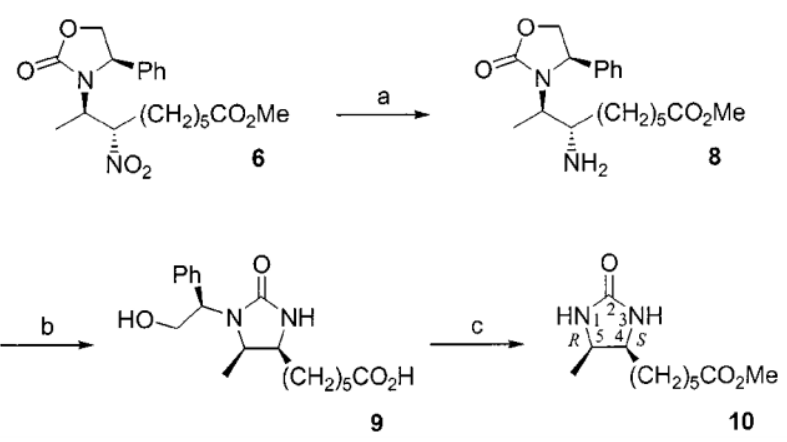

Scheme 5. Preparation of imidazolidinone 10 from nitro compound 6; reagents and conditions: (a) $\mathrm{HCO}_{2} \mathrm{NH}_{4}, \mathrm{Pd} / \mathrm{C}, \mathrm{MeOH}$, 3 days, 64\%; (b) $\mathrm{KOH}, \mathrm{MeOH}, 16$ h, reflux, $89 \%$; (c) $\mathrm{H}_{2}, \mathrm{Pd}(\mathrm{OH})_{2} / \mathrm{C}, \mathrm{MeOH}, \mathrm{H}_{2} \mathrm{SO}_{4}$ (cat.), 2 days, $64 \%$

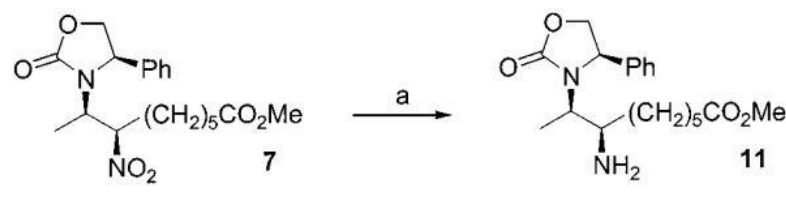

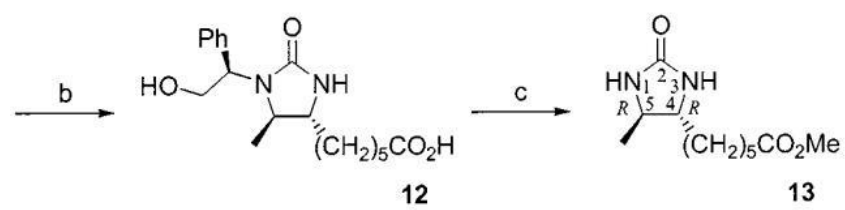

Scheme 6. Preparation of imidazolidinone 13 from nitro compound 7; reagents and conditions: (a) $\mathrm{HCO}_{2} \mathrm{NH}_{4}, \mathrm{Pd} / \mathrm{C}, \mathrm{MeOH}$, 3 days, $72 \%$; (b) $\mathrm{KOH}, \mathrm{MeOH}, 16$ h, reflux, $89 \%$; (c) $\mathrm{H}_{2}, \mathrm{Pd}(\mathrm{OH})_{2} / \mathrm{C}, \mathrm{MeOH}, \mathrm{H}_{2} \mathrm{SO}_{4}$ (cat.), 2 days, $63 \%$

The structures of the four imidazolidinones prepared were determined (a) by comparison of their spectroscopical properties (Table 2) with those of a sample prepared by esterification of authentic d-dethiobiotin ( $\mathrm{MeOH}, \mathrm{H}_{2} \mathrm{SO}_{4}, 12 \mathrm{~h}$, room temp., $91 \%$ ) and (b) by comparison of their optical rotation values (Table 3) with those reported in the literature. ${ }^{[7]}$

Table 2. Spectroscopic characteristics of imidazolidinones $\mathbf{1 0}$ and $\mathbf{1 3}$<smiles>CC(C)=O</smiles>

\begin{tabular}{|c|c|c|c|c|c|c|}
\hline & $\begin{array}{l}\mathrm{H}_{4} \\
\delta(\mathrm{ppm})\end{array}$ & $\begin{array}{l}\mathrm{H}_{5} \\
\delta(\mathrm{ppm})\end{array}$ & $\begin{array}{l}J\left(\mathrm{H}_{4}-\mathrm{H}_{5}\right) \\
(\mathrm{Hz})\end{array}$ & $\begin{array}{l}\mathrm{C}_{4} \\
\delta(\mathrm{ppm})\end{array}$ & $\begin{array}{l}\mathrm{C}_{5} \\
\delta(\mathrm{ppm})\end{array}$ & $\begin{array}{l}\mathrm{CH}_{3} \mathrm{CH} \\
\delta(\mathrm{ppm})\end{array}$ \\
\hline $\begin{array}{l}10(\text { cis }) \\
13 \text { (trans) }\end{array}$ & $\begin{array}{l}3.70-3.60 \\
3.13\end{array}$ & $\begin{array}{l}3.83 \\
3.33\end{array}$ & $\begin{array}{l}7.7 \\
5.5\end{array}$ & $\begin{array}{l}55.8 \\
60.2\end{array}$ & $\begin{array}{l}51.3 \\
54.0\end{array}$ & $\begin{array}{l}15.5 \\
21.0\end{array}$ \\
\hline
\end{tabular}


Table 3. Optical rotations of imidazolidinones 10, ent-10, 13, ent-13

\begin{tabular}{lcrrrl}
\hline & 10 & ent-10 & 13 & ent-13 & $\begin{array}{l}\text { D-dethiobiotin } \\
\text { methyl ester }{ }^{[7]}\end{array}$ \\
\hline$[\alpha]_{\mathrm{D}}$ & -2.3 & +2.1 & +42.5 & -39.7 & +2.6 \\
$c\left(\mathrm{CHCl}_{3}\right)$ & 0.865 & 0.74 & 1.15 & 1.28 & 2.00 \\
\hline
\end{tabular}

The product ent-10 was found to be identical to d-dethiobiotin methyl ester and thus its absolute configuration is $(4 R, 5 S)$. Hence, the absolute configurations of the three other diastereomers 10, 13, and ent-13 are $(4 S, 5 R),(4 R, 5 R)$, and $(4 S, 5 S)$, respectively. The $(R)$ absolute configuration observed for carbon 5 in compounds $\mathbf{1 0}$ and $\mathbf{1 3}$, which are both derived from 4phenyloxazolidin-2-one $(R)-\mathbf{3}$, was expected on the basis of our previous study of the conjugate addition to nitroalkenes. ${ }^{[4,5]}$ In conclusion, the highly stereoselective conjugate addition of 4-phenyloxazolidin-2-one to nitroalkene $\mathbf{2}$ served as the key step in the synthesis of the methyl esters of natural d-dethiobiotin and its diastereomers. The action of the unnatural isomers on biotin biosynthesis will be evaluated.

\section{Experimental Section}

General: THF was freshly distilled from sodium benzophenone ketyl. — TLC: Silica Gel 60F 254 plates (Merck), with detection by $\mathrm{UV}$ light or with an acidic solution of ninhydrin in $t \mathrm{BuOH}$ or with a solution of phosphomolybdic acid in EtOH. — Column chromato- graphy: 40 - $63 \mu \mathrm{m}$ Merck Silica Gel. — IR: Perkin —Elmer 2000.—Optical rotations: Perkin-Elmer 341 micropolarimeter. Melting points (uncorrected): Büchi 535. — NMR: Bruker AM 300 (300.13 and $75.47 \mathrm{MHz}$ for ${ }^{1} \mathrm{H}$ and ${ }^{13} \mathrm{C}$, respectively). - MS: Finnegan- Mat $4600(70 \mathrm{eV})$.

Methyl 7-Bromoheptanoate: Concentrated sulfuric acid $(20 \mathrm{~g})$ was slowly added to methanol $(25 \mathrm{~mL})$ cooled at $0^{\circ} \mathrm{C}$. 7 Bromoheptanenitrile $(8.45 \mathrm{~g}, 44.5 \mathrm{mmol})$ was then added and the mixture was heated at reflux for $40 \mathrm{~h}$. After cooling to room temp., cold water $(50 \mathrm{~mL})$ was added, the organic phase was separated and the aqueous phase was extracted with ether $(4 \times$ $50 \mathrm{~mL}$ ). The combined organic phases were then dried with magnesium sulfate. After filtration and concentration in vacuo, chromatography on silica gel (95:5 pentane/AcOEt) afforded methyl 7-bromoheptanoate $(6.49 \mathrm{~g}, 66 \%)$ as a colorless oil. ${ }^{1} \mathrm{H} \mathrm{NMR}\left(\mathrm{CDCl}_{3}\right): \delta=1.40\left(\mathrm{~m}, 4 \mathrm{H}, \mathrm{CH}_{2} \mathrm{CH}_{2} \mathrm{CH}_{2} \mathrm{CH}_{2} \mathrm{Br}\right), 1.61\left(\mathrm{~m}, 2 \mathrm{H}, \mathrm{CH}_{2} \mathrm{CH}_{2} \mathrm{CO}\right), 1.83\left(\mathrm{~m}, 2 \mathrm{H}, \mathrm{CH}_{2} \mathrm{CH}_{2} \mathrm{Br}\right), 2.29(\mathrm{t}, J=$ $\left.7.5 \mathrm{~Hz}, 2 \mathrm{H}, \mathrm{CH}_{2} \mathrm{CO}\right), 3.37\left(\mathrm{t}, J=6.8 \mathrm{~Hz}, 2 \mathrm{H}, \mathrm{CH}_{2} \mathrm{Br}\right), 3.63\left(\mathrm{~s}, 3 \mathrm{H}, \mathrm{OCH}_{3}\right) .-{ }^{13} \mathrm{C} \mathrm{NMR}\left(\mathrm{CDCl}_{3}\right): \delta=24.5,27.5,28.0,32.3$, 33.4, $33.6(\mathrm{C} 2-\mathrm{C} 7), 51.2\left(\mathrm{CH}_{3} \mathrm{O}\right), 173.8(\mathrm{C} 1)$.

Methyl 7-Iodoheptanoate: Sodium iodide ( $3.36 \mathrm{~g}, 22.4 \mathrm{mmol})$ was added to a solution of methyl 7-bromoheptanoate (1.00 $\mathrm{g}, 4.50 \mathrm{mmol})$ in acetone $(15 \mathrm{~mL})$. The suspension was heated at reflux for $30 \mathrm{~h}$ under argon, in the dark. After cooling to room temp., aqueous sodium thiosulfate $(0.1 \mathrm{~N}, 20 \mathrm{~mL})$ was added, the organic phase was separated and the aqueous phase was extracted with diethyl ether $(3 \times 20 \mathrm{~mL})$. The combined organic phases were washed with water $(2 \times 10 \mathrm{~mL})$, then dried with magnesium sulfate. After filtration and concentration in vacuo, methyl 7-iodoheptano- ate $(1.14 \mathrm{~g}, 94 \%)$ was obtained as a colorless oil, which was used in the next step without further purification. $-{ }^{1} \mathrm{H}$ NMR $\left(\mathrm{CDCl}_{3}\right): 1.80\left(\mathrm{~m}, 2 \mathrm{H}, \mathrm{CH}_{2} \mathrm{CH}_{2} \mathrm{I}\right)$, $2.29\left(\mathrm{t}, J=7.5 \mathrm{~Hz}, 2 \mathrm{H}, \mathrm{CH}_{2} \mathrm{CO}\right), 3.16\left(\mathrm{t}, J=7.0 \mathrm{~Hz}, 2 \mathrm{H}, \mathrm{CH}_{2} \mathrm{I}\right), 3.65\left(\mathrm{~s}, 3 \mathrm{H}, \mathrm{OCH}_{3}\right) .-{ }^{13} \mathrm{C} \mathrm{NMR}\left(\mathrm{CDCl}_{3}\right): \delta=6.6\left(\mathrm{CH}_{2} \mathrm{I}\right)$, $24.4,27.8,29.9,33.0,33.7(\mathrm{C} 2-\mathrm{C} 6), 51.2\left(\mathrm{CH}_{3} \mathrm{O}\right), 173.8(\mathrm{C} 1)$.

Methyl 7-Nitroheptanoate (4): Silver nitrite $(6.68 \mathrm{~g}, 43.4 \mathrm{mmol})$ was added to a solution of methyl 7-iodoheptanoate (5.86 $\mathrm{g}, 21.7 \mathrm{mmol})$ in diethyl ether $(75 \mathrm{~mL})$. The suspension was stirred for $3 \mathrm{~d}$ under argon, in the dark. The solid phase was removed by filtration and washed with methanol $(2 \times 20 \mathrm{~mL})$. The combined organic phases were filtered to remove residual solids. After filtration and concentration in vacuo, chromatography on silica gel (90:10 pentane/AcOEt) afforded methyl 7-nitroheptanoate $(3.28 \mathrm{~g}, 80 \%)$ as a colorless oil. $-{ }^{1} \mathrm{H}$ NMR $\left(\mathrm{CDCl}_{3}\right): \delta=1.35-1.44\left(\mathrm{~m}, 4 \mathrm{H}, \mathrm{CH}_{2} \mathrm{CH}_{2} \mathrm{CH}_{2} \mathrm{CH}_{2} \mathrm{NO}_{2}\right)$, 1.57-1.65 (m, $2 \mathrm{H}, \mathrm{CH}_{2} \mathrm{CH}_{2} \mathrm{CO}$ ), $2.01\left(\mathrm{~m}, 2 \mathrm{H}, \mathrm{CH}_{2} \mathrm{CH}_{2} \mathrm{NO}_{2}\right), 2.30$ (t, J = 7.3 Hz, $2 \mathrm{H}, \mathrm{CH}_{2} \mathrm{CO}$ ), 3.65 (s, 3H, OCH $\mathrm{OCH}_{3}, 4.36$ $\left(\mathrm{t}, J=7.1 \mathrm{~Hz}, 2 \mathrm{H}, \mathrm{CH}_{2} \mathrm{NO}_{2}\right) .-{ }^{13} \mathrm{C}$ NMR $\left(\mathrm{CDCl}_{3}\right): \delta=24.0,25.4,26.7,27.8(\mathrm{C} 3-\mathrm{C} 6), 33.4(\mathrm{C} 2), 50.8\left(\mathrm{CH}_{3} \mathrm{O}\right), 75.0$ $\left(\mathrm{CH}_{2} \mathrm{NO}_{2}\right), 173.2(\mathrm{C} 1)$.

Methyl 8-Hydroxy-7-nitrononanoate (5): To a solution of nitroester $4(5.2 \mathrm{~g}, 27.5 \mathrm{mmol})$ in methanol (50 $\mathrm{mL}) \mathrm{cooled}$ at $0^{\circ} \mathrm{C}$ were added successively a solution of potassium hydroxide $(1.54 \mathrm{~g}, 27.5 \mathrm{mmol})$ in methanol $(25 \mathrm{~mL})$ and acetaldehyde $(1.55 \mathrm{~mL}, 27.5 \mathrm{mmol})$. Additional acetaldehyde was added after $4 \mathrm{~h}(1.55 \mathrm{~mL})$, and after $11 \mathrm{~h}(1.55 \mathrm{~mL})$. After $19 \mathrm{~h}$, acetic acid was added. After concentration in vacuo, water $(2 \mathrm{~mL})$ was added and the aqueous phase was extracted with diethyl ether $(4 \times 10 \mathrm{~mL})$. The combined organic phases were then dried with magnesium sulfate. After filtration and concentration in vacuo, chromatography on silica gel (70:30 pentane/AcOEt) afforded nitroalcohol 5 (5.37 g, 84\%, mixture of two diastereomers) as a colorless oil. IR (film): $\bar{v}=3469 \mathrm{~cm}^{-1}$ (broad, OH), 2938, 2864, $1737(\mathrm{C}=\mathrm{O}), 1550,1371 .{ }^{1} \mathrm{H}$ NMR $\left(\mathrm{CDCl}_{3}\right): \delta=1.28\left(\mathrm{~d}, J=6.7 \mathrm{~Hz}, 3 \mathrm{H}, \mathrm{CHCH}_{3}\right.$, diastereomer 1), $1.28\left(\mathrm{~d}, J=6.6 \mathrm{~Hz}, 3 \mathrm{H}, \mathrm{CHCH}_{3}\right.$, diastereomer 2), 1.30$1.40\left(\mathrm{~m}, 4 \mathrm{H}, \mathrm{CH}_{2} \mathrm{CH}_{2} \mathrm{CH}_{2} \mathrm{CH}_{2} \mathrm{CO}\right), 1.57-1.63\left(\mathrm{~m}, 2 \mathrm{H}, \mathrm{CH}_{2} \mathrm{CH}_{2} \mathrm{CO}\right), 1.77-1.82(\mathrm{~m}, 1 \mathrm{H}, \mathrm{CHHCHNO}), 1.97-2.07(\mathrm{~m}, 1$ $\mathrm{H}, \mathrm{CHHCHNO} 2), 2.21(\mathrm{~d}, J=7.0 \mathrm{~Hz}, 1 \mathrm{H}, \mathrm{OH}$, diastereomer 1), $2.28($ broad s, $1 \mathrm{H}, \mathrm{OH}$, diastereomer 2), $2.30(\mathrm{t}, J=7.3 \mathrm{~Hz}$, $\left.2 \mathrm{H}, \mathrm{CH}_{2} \mathrm{CO}\right), 3.66\left(\mathrm{~s}, 3 \mathrm{H}, \mathrm{OCH}_{3}\right), 4.10(\mathrm{~m}, 1 \mathrm{H}, \mathrm{CHOH}$, diastereomer 1), $4.18(\mathrm{~m}, 1 \mathrm{H}, \mathrm{CHOH}$, diastereomer 2), $4.36-4.41$ 
$(\mathrm{m}, 1 \mathrm{H}, \mathrm{CHNO} 2) .-{ }^{13} \mathrm{C} \mathrm{NMR}\left(\mathrm{CDCl}_{3}\right): \delta=18.8$ (C9, diastereomer 1), $19.4(\mathrm{C} 9$, diastereomer 2), $24.1,25.0($ diastereomer 1), 25.3 (diastereomer 2), 28.0 (diastereomer 1), 28.1 (diastereomer 2), 29.6 (C3- C6), 33.4 (C2), 51.3 (CH $\mathrm{CH}_{3} \mathrm{O}, 68.2(\mathrm{C} 8)$, 92.9 (C7, diastereomer 1), 94.0 (C7, diastereomer 2), 173.0 (C1). $-\mathrm{C}_{10} \mathrm{H}_{19} \mathrm{NO}_{5}$ (233.3): calcd. C 51.49, H 8.21, $\mathrm{N}$ 6.01; found C 51.63, H 8.11, N 5.91.

Methyl 8-Acetoxy-7-nitrononanoate: To a solution of nitroalcohol 5 (5.2 g, $22.3 \mathrm{mmol})$ in anhydrous diethyl ether (150 $\mathrm{mL}$ ) was added a solution of DMAP (4-dimethylaminopyridine, $1.36 \mathrm{~g}, 11.2 \mathrm{mmol}$ ) in acetic anhydride (21.1 mL). After stirring for $16 \mathrm{~h}$ at room temp., saturated aqueous sodium hydrogen carbonate $(30 \mathrm{~mL})$ was added. Solid sodium hydrogen carbonate was then added until gas evolution ceased. The phases were separated and the aqueous phase was extracted with diethyl ether $(2 \times 50 \mathrm{~mL})$. The combined organic phases were then dried with magnesium sulfate. Filtration and concentration in vacuo afforded an oil $(4.5 \mathrm{~g})$ containing mostly the expected nitro acetate and a little nitroalkene 2 . The crude product was used as such in the next step. Characteristics of the major diastereomer: $\left.-{ }^{1} \mathrm{H} N \mathrm{NR}_{(\mathrm{CDCl}}\right): \delta=1.29(\mathrm{~d}, J=$ $6.5 \mathrm{~Hz}, 3 \mathrm{H}, \mathrm{CHCH}), 1.24-1.38(\mathrm{~m}, 4 \mathrm{H}, \mathrm{CH} \mathrm{CH} \mathrm{CH} \mathrm{CH} \mathrm{CO}), 1.59-1.66\left(\mathrm{~m}, 2 \mathrm{H}, \mathrm{CH}_{2} \mathrm{CH}_{2} \mathrm{CO}\right), 1.66-1.68(\mathrm{~m}, 1 \mathrm{H}$, $\left.\mathrm{CHHCHNO}_{2}\right), 1.98-2.02\left(\mathrm{~m}, 1 \mathrm{H}, \mathrm{CH} H \mathrm{CHNO}\right.$ ), $2.01\left(\mathrm{~s}, 3 \mathrm{H}, \mathrm{CH}_{3} \mathrm{CO}\right), 2.29\left(\mathrm{t}, \mathrm{J}=7.4 \mathrm{~Hz}, 2 \mathrm{H}, \mathrm{CH} \mathrm{CO}_{2}, 3.66(\mathrm{~s}, 3 \mathrm{H}\right.$, $\left.\mathrm{OCH}_{3}\right), 4.52(\mathrm{ddd}, J=11.3,8.2,3.2 \mathrm{~Hz}, 1 \mathrm{H}, \mathrm{CHNO}), 5.25\left(\mathrm{qd}, J=8.2,6.5 \mathrm{~Hz}, 1 \mathrm{H}, \mathrm{CHCH}_{3}\right) .-{ }^{13} \mathrm{C} \mathrm{NMR}\left(\mathrm{CDCl}_{3}\right): \delta=$ 16.3 (C9), $20.5\left(\mathrm{CH}_{3} \mathrm{CO}\right), 24.1,24.9,28.0,29.2$ (C3-C6), $33.4(\mathrm{C} 2), 51.1\left(\mathrm{CH}_{3} \mathrm{O}\right), 69.8(\mathrm{C} 8), 90.7(\mathrm{C} 7), 169.3(\mathrm{CH} 3 \mathrm{CO})$, $173.4(\mathrm{C} 1)$.

Methyl 7-Nitronon-7-enoate (2): To a solution of the crude product obtained above (4.5 g) in dichloromethane (20 mL) were added DMAP $(6.8 \mathrm{~g}, 55.6 \mathrm{mmol})$ and basic alumina $(7.5 \mathrm{~g}$, activity I). The reaction mixture was heated at reflux for 4 $\mathrm{h}$, cooled to room temp., and then filtered over a short pad of celite. After concentration in vacuo, chromatography on silica gel (85:15 pentane/AcOEt) afforded nitroalkene $2(3.00 \mathrm{~g}, 63 \%$ from nitro alcohol 5) as a 90:10 mixture of $(E)-$ and $(Z)$ isomers. Characteristics of the major isomer: ${ }^{1} \mathrm{H}$ NMR $\left(\mathrm{CDCl}_{3}\right): \delta=1.31-1.39\left(\mathrm{~m}, 2 \mathrm{H}, \mathrm{CH}_{2} \mathrm{CH}_{2} \mathrm{CH}_{2} \mathrm{CO}\right), 1.45-1.52(\mathrm{~m}, 2$ $\left.\mathrm{H}, \mathrm{CH}_{2} \mathrm{CH}_{2} \mathrm{C}=\right)$, $1.58-1.67\left(\mathrm{~m}, 2 \mathrm{H}, \mathrm{CH}_{2} \mathrm{CH}_{2} \mathrm{CO}\right), 1.87\left(\mathrm{~d}, J=7.4 \mathrm{~Hz}, 3 \mathrm{H}, \mathrm{CH}_{3} \mathrm{CH}=\right), 2.30(\mathrm{t}, \mathrm{J}=7.4 \mathrm{~Hz}, 2 \mathrm{H}, \mathrm{CH} \mathrm{CO})$, $2.58\left(\mathrm{t}, J=7.5 \mathrm{~Hz}, 2 \mathrm{H}, \mathrm{CH}_{2} \mathrm{C}=\right), 3.66\left(\mathrm{~s}, 3 \mathrm{H}, \mathrm{OCH}_{3}\right), 7.16\left(\mathrm{q}, J=7.4 \mathrm{~Hz}, 1 \mathrm{H}, \mathrm{CH}_{3} \mathrm{CH}=\right) .-{ }^{13} \mathrm{C} \mathrm{NMR}\left(\mathrm{CDCl}_{3}\right): \delta=14.0$ (C9), 24.3, 25.6, 27.0, 28.3 (C3-C6), 33.6 (C2), 51.1 ( $\left.\mathrm{CH}_{3} \mathrm{O}\right), 130.9(\mathrm{C} 8), 152.4(\mathrm{C} 7), 173.6(\mathrm{C} 1)$.

Nitrooxazolidinones 6 and 7: A mixture of $(R)$-4-phenyloxazolidin- 2-one (989 mg, 6.06 mmol), potassium tert-butoxide $(680 \mathrm{mg}, 6.06 \mathrm{mmol})$ and 18 -crown-6 $(123 \mathrm{mg}, 0.47 \mathrm{mmol})$ in THF $(35 \mathrm{~mL})$ was stirred under argon at $0^{\circ} \mathrm{C}$ for $20 \mathrm{~min}$, and then cooled to $-78^{\circ} \mathrm{C}$. A solution of nitroalkene $2(1.00 \mathrm{~g}, 4.66 \mathrm{mmol})$ in THF $(5 \mathrm{~mL})$ cooled at $-78^{\circ} \mathrm{C}$ was added dropwise. After $45 \mathrm{~min}$ at $-78^{\circ} \mathrm{C}$, no more nitroalkene remained in the reaction mixture. Acetic acid $(0.67 \mathrm{~mL}, 11.65 \mathrm{mmol}) \mathrm{was}$ added and the flask was allowed to warm to room temp. Water $(20 \mathrm{~mL})$ and dichloromethane $(100 \mathrm{~mL})$ were added, the phases were separated and the aqueous phase was extracted with dichloromethane $(3 \times 50 \mathrm{~mL})$. The combined organic phases were then dried with magnesium sulfate. After filtration and concentration in vacuo, the yellow oil obtained was dissolved in dichloromethane $(100 \mathrm{~mL})$ and triethylamine $(4 \mathrm{~mL})$ was added. After $5 \mathrm{~d}$ at room temp., the solution was concentrated in vacuo. Chromatography on silica gel (75:25 pentane/AcOEt) afforded the two nitro compounds $6(0.6775 \mathrm{~g}, 38 \%$, less polar) as a slightly yellow oil and $7(0.7824 \mathrm{~g}, 45 \%$, more polar) as a slightly yellow solid.

6: $[\alpha]_{\mathrm{D}}^{28}=-33.3\left(c=0.650, \mathrm{CH}_{3} \mathrm{OH}\right) .-\mathrm{IR}(\mathrm{film}): \bar{v}=2948 \mathrm{~cm}^{-1}, 2864,1748,1552,1417,1215,1039 .-{ }^{1} \mathrm{H} \mathrm{NMR}\left(\mathrm{CDCl}_{3}\right)$ : $\delta=1.12\left(\mathrm{~d}, J=6.8 \mathrm{~Hz}, 3 \mathrm{H}, \mathrm{CH}_{3} \mathrm{CH}\right), 1.18-1.33\left(\mathrm{~m}, 4 \mathrm{H}, \mathrm{CH}_{2} \mathrm{CH}_{2} \mathrm{CH}_{2} \mathrm{CH}_{2} \mathrm{CO}\right), 1.53-1.64\left(\mathrm{~m}, 3 \mathrm{H}, \mathrm{CH} \mathrm{CH}_{2} \mathrm{CO}_{2}\right.$ $\left.\mathrm{CHHCHNO}_{2}\right), 1.67-1.80\left(\mathrm{~m}, 1 \mathrm{H}, \mathrm{CH} H \mathrm{CHNO}_{2}\right), 2.26\left(\mathrm{t}, J=7.4 \mathrm{~Hz}, 2 \mathrm{H}, \mathrm{CH}_{2} \mathrm{CO}\right), 3.62\left(\mathrm{~m}, 1 \mathrm{H}, \mathrm{CHCH}_{3}\right), 3.65(\mathrm{~s}, 3 \mathrm{H}$, $\left.\mathrm{OCH}_{3}\right), 4.21(\mathrm{dd}, J=8.5,6.9 \mathrm{~Hz}, 1 \mathrm{H}, \mathrm{OCHHCHN}), 4.62(\mathrm{t}, J=8.5 \mathrm{~Hz}, 1 \mathrm{H}, \mathrm{OCH} H \mathrm{CHN}), 4.71(\mathrm{dd}, J=8.5,6.9 \mathrm{~Hz}, 1 \mathrm{H}$, $\mathrm{CHPh}), 5.15$ (ddd, $\left.J=10.7,8.5,3.3 \mathrm{~Hz}, 1 \mathrm{H}, \mathrm{CHNO}_{2}\right), 7.30-7.43(\mathrm{~m}, 5 \mathrm{H}, \mathrm{Ar}-\mathrm{H}) .-{ }^{13} \mathrm{C} \mathrm{NMR}\left(\mathrm{CDCl}_{3}\right) \delta=12.9(\mathrm{C} 9), 24.1$, 24.9, 27.9, $30.4(\mathrm{C} 3-\mathrm{C} 6), 33.5(\mathrm{C} 2), 51.2\left(\mathrm{CH}_{3} \mathrm{O}\right), 51.8(\mathrm{C} 8), 59.8(\mathrm{CHPh}), 70.2(\mathrm{OCH} \mathrm{CHN}), 89.8(\mathrm{C} 7), 127.2,129.2,129.4$ (Ar-C), 137.4 (ipso-Ar-C), 157.2 (NCOO), 173.6 (C1). - $\mathrm{C}_{19} \mathrm{H}_{26} \mathrm{~N}_{2} \mathrm{O}_{6}$ (378.4): calcd. C 60.30, H 6.93, N 7.40; found C 60.35, H 6.83, N 7.52.

ent-6: Same characteristics as 6 except for the optical rotation. $[\alpha]_{\mathrm{D}}^{28}=+37.0\left(c=0.732, \mathrm{CH}_{3} \mathrm{OH}\right)$.

7: $[\alpha]^{29}=-78.9\left(c=1.041, \mathrm{CH}_{3} \mathrm{OH}\right) .-\mathrm{IR}(\mathrm{film}): \bar{v}=2945 \mathrm{~cm}^{-1}, 1756,1548,1242,1032 .-{ }^{1} \mathrm{H} \mathrm{NMR}\left(\mathrm{CDCl}_{3}\right): \delta=1.07(\mathrm{~d}$, $\left.J=7.0 \mathrm{~Hz}, 3 \mathrm{H}, \mathrm{CH}_{3} \mathrm{CH}\right), 1.19-1.37\left(\mathrm{~m}, 4 \mathrm{H}, \mathrm{CH}_{2} \mathrm{CH}_{2} \mathrm{CH}_{2} \mathrm{CH}_{2} \mathrm{CO}\right), 1.51-1.76\left(\mathrm{~m}, 4 \mathrm{H}, \mathrm{CH}_{2} \mathrm{CH}_{2} \mathrm{CO}_{2} \mathrm{CH}_{2} \mathrm{CHNO}_{2}\right), 2.22$ $\left(\mathrm{t}, J=7.4 \mathrm{~Hz}, 2 \mathrm{H}, \mathrm{CH}_{2} \mathrm{CO}\right), 3.65\left(\mathrm{~s}, 3 \mathrm{H}, \mathrm{OCH}_{3}\right), 3.72\left(\mathrm{~m}, 1 \mathrm{H}, \mathrm{CHCH}_{3}\right), 4.08(\mathrm{~m}, 1 \mathrm{H}, \mathrm{OCHHCHN}), 4.50(\mathrm{t}, J=8.5 \mathrm{~Hz}, 1$ $\mathrm{H}, \mathrm{OCH} H \mathrm{CHN}), 4.75(\mathrm{t}, J=7.8 \mathrm{~Hz}, 1 \mathrm{H}, \mathrm{CHPh}), 5.16\left(\mathrm{td}, J=10.8,3.5 \mathrm{~Hz}, 1 \mathrm{H}, \mathrm{CHNO}_{2}\right), 7.22-7.37(\mathrm{~m}, 5 \mathrm{H}, \mathrm{Ar}-\mathrm{H})$. ${ }^{13} \mathrm{C} \mathrm{NMR}\left(\mathrm{CDCl}_{3}\right) \delta=13.6(\mathrm{C} 9), 24.1,25.0,28.0,30.3(\mathrm{C} 3-\mathrm{C} 6), 33.4(\mathrm{C} 2), 51.2\left(\mathrm{CH}_{3} \mathrm{O}\right), 52.6(\mathrm{C} 8), 59.6(C H P h), 70.0$ $\left(\mathrm{OCH}_{2} \mathrm{CHN}\right), 89.1$ (C7), 127.3, 129.0, 129.3 (Ar-C), 137.0 (ipso-Ar-C), $157.3(\mathrm{NCOO}), 173.5(\mathrm{C} 1) .-\mathrm{C}_{19} \mathrm{H}_{26} \mathrm{~N}_{2} \mathrm{O}_{6}(378.4)$ : calcd. C 60.30, H 6.93, N 7.40; found C 60.53, H 6.92, N 7.24.

ent-7: Same characteristics as 7 except for the optical rotation. $[\alpha]_{\mathrm{D}}^{21}=+75.2\left(c=0.938, \mathrm{CH}_{3} \mathrm{OH}\right)$. 
Aminooxazolidinone 8: To a solution of nitro compound $6(0.676 \mathrm{~g}, 1.79 \mathrm{mmol})$ in methanol $(60 \mathrm{~mL})$ were added palladium on carbon $(10 \%, 0.300 \mathrm{~g})$ and anhydrous ammonium formate $(0.563 \mathrm{~g}, 8.93 \mathrm{mmol})$. After stirring for $3 \mathrm{~d}$ at room temp., the suspension was filtered through a short pad of celite which was then washed with methanol. After concentration in vacuo, chromatography on silica gel (95:3:0.5 $\left.\mathrm{MeOH} / \mathrm{CH}_{2} \mathrm{Cl}_{2} / \mathrm{Et}_{3} \mathrm{~N}\right)$ afforded amine $8(0.399 \mathrm{~g}, 64 \%)$ as a viscous, colorless oil. 8: $[\alpha]^{26}=-28.5\left(c=0.68, \mathrm{CH}_{3} \mathrm{OH}\right) .-\mathrm{IR}(\mathrm{film}): \bar{v}=3379 \mathrm{~cm}^{-1}, 2937,2860,1736(\mathrm{C}=\mathrm{O}), 1591,1222,705 .-{ }^{1} \mathrm{H} \mathrm{NMR}(\mathrm{CDCl})$ : $\delta=1.10\left(\mathrm{~d}, J=7.0 \mathrm{~Hz}, 3 \mathrm{H}, \mathrm{CH}_{3} \mathrm{CH}\right), 1.13-1.44\left(\mathrm{~m}, 6 \mathrm{H}, \mathrm{CH}_{2} \mathrm{CH}_{2} \mathrm{CH}_{2} \mathrm{CHNH}_{2}\right), 1.48\left(\mathrm{~m}, 2 \mathrm{H}, \mathrm{CH}_{2} \mathrm{CH} \mathrm{CO}_{2} \mathrm{CO}, 2.20(\mathrm{t}, J=7.3\right.$ $\left.\mathrm{Hz}, 2 \mathrm{H}, \mathrm{CH}_{2} \mathrm{CO}\right), 3.18\left(\mathrm{~m}, 1 \mathrm{H}, \mathrm{CHNH}\right.$ ), $3.31\left(\mathrm{~m}, 1 \mathrm{H}, \mathrm{CHCH}_{3}\right), 3.61\left(\mathrm{~s}, 3 \mathrm{H}, \mathrm{OCH}_{3}\right), 4.13(\mathrm{dd}, J=8.5,6.6 \mathrm{~Hz}, 1 \mathrm{H}$, OCHHCHN), 4.32 (broad s, $\left.2 \mathrm{H}, \mathrm{NH}_{2}\right), 4.62(\mathrm{t}, J=8.5 \mathrm{~Hz}, 1 \mathrm{H}, \mathrm{OCH} H \mathrm{CHN}), 4.88(\mathrm{dd}, J=8.5,6.6 \mathrm{~Hz}, 1 \mathrm{H}, \mathrm{CHPh}), 7.30-$ $7.38(\mathrm{~m}, 5 \mathrm{H}, \mathrm{Ar}-\mathrm{H}) .-{ }^{13} \mathrm{C} \mathrm{NMR}\left(\mathrm{CDCl}_{3}\right) \delta=11.6(\mathrm{C} 9), 24.4,25.2,28.4,32.9(\mathrm{C} 3-\mathrm{C} 6), 33.6(\mathrm{C} 2), 51.1\left(\mathrm{CH} \mathrm{C}_{3} \mathrm{O}\right), 53.0(\mathrm{C} 7)$, $54.8(\mathrm{C} 8), 60.6(\mathrm{CHPh}), 70.4\left(\mathrm{OCH}_{2} \mathrm{CHN}\right), 127.0,128.9,129.0(\mathrm{Ar}-\mathrm{C}), 138.8$ (ipso-Ar-C), 158.5 (NCOO), $173.7(\mathrm{C} 1)$. $\mathrm{C}_{19} \mathrm{H}_{30} \mathrm{~N}_{2} \mathrm{O}_{5}\left(8+\mathrm{H}_{2} \mathrm{O}, 366.2\right)$ : calcd. C 62.27, H 8.25, N 7.64; found C 62.42, H 7.75, N 7.26.

ent-8: Same characteristics as $\mathbf{8}$ except for the optical rotation. $[\alpha]_{\mathrm{D}}^{27}=+27.9\left(c=0.84, \mathrm{CH}_{3} \mathrm{OH}\right)$

Aminooxazolidinone 11: The same procedure described for the preparation of amine 8 was applied to nitro compound 7 $(0.75 \mathrm{~g}, 1.98 \mathrm{mmol})$, and afforded amine $11(0.496 \mathrm{~g}, 72 \%)$ as a viscous, slightly yellow oil. 11: $[\alpha]^{27}=-27.5(c=0.86, \mathrm{CH}$ OH). - IR (film): $\bar{v}=3382$ (broad) $\mathrm{cm}^{-1}, 2939,2860,1733(\mathrm{C}=\mathrm{O}), 1603,1223,704 .-{ }^{1} \mathrm{H} \mathrm{NMR}^{2}\left(\mathrm{CDCl}_{3}\right): \delta=0.78(\mathrm{~d}, J=$ $\left.6.8 \mathrm{~Hz}, 3 \mathrm{H}, \mathrm{CH}_{3} \mathrm{CH}\right), 1.11-1.48\left(\mathrm{~m}, 8 \mathrm{H}, \mathrm{CH}_{2} \mathrm{CH}_{2} \mathrm{CH}_{2} \mathrm{CH}_{2} \mathrm{CHNH}_{2}\right), 2.22(\mathrm{t}, J=7.3 \mathrm{~Hz}, 2 \mathrm{H}, \mathrm{CH} 2 \mathrm{CO}), 3.09(\mathrm{~m}, 1 \mathrm{H}$, $\left.\mathrm{CHNH}_{2}\right), 3.41\left(\mathrm{~m}, 1 \mathrm{H}, \mathrm{CHCH}_{3}\right), 3.58\left(\mathrm{~s}, 3 \mathrm{H}, \mathrm{OCH}_{3}\right), 4.06(\mathrm{dd}, J=8.2,6.9 \mathrm{~Hz}, 1 \mathrm{H}, \mathrm{OCHHCHN}), 4.33\left(\mathrm{broad} \mathrm{s}, 2 \mathrm{H}, \mathrm{NH}_{2}\right)$, $4.55(\mathrm{t}, J=8.2 \mathrm{~Hz}, 1 \mathrm{H}, \mathrm{OCH} H \mathrm{CHN}), 4.90(\mathrm{dd}, J=8.2,6.9 \mathrm{~Hz}, 1 \mathrm{H}, \mathrm{CHPh}), 7.30\left(\mathrm{~m}, 5 \mathrm{H}, \mathrm{Ar}_{-\mathrm{H}}\right) .-{ }^{13} \mathrm{C} \mathrm{NMR}(\mathrm{CDCl}): \delta=$ 15.9 (C9), 24.4, 24.9, 28.9, $33.0(\mathrm{C} 3-\mathrm{C} 6), 33.7(\mathrm{C} 2), 51.2\left(\mathrm{CH}_{3} \mathrm{O}\right), 53.3(\mathrm{C} 7), 54.2(\mathrm{C} 8), 58.9(\mathrm{CHPh}), 70.4\left(\mathrm{OCH} \mathrm{CHN}_{2}\right.$, 127.3, 128.8, 128.9 (Ar-C), 139.3 (ipso- Ar-C), 158.9 (NCOO), 173.9 (C1).

ent-11: Same characteristics as 11 except for the optical rotation. $[\alpha]_{\mathrm{D}}^{27}=+28.1\left(c=1.63, \mathrm{CH}_{3} \mathrm{OH}\right)$

Imidazolidinone 9: A solution of amine $8(0.430 \mathrm{~g}, \mathrm{mmol})$ and potassium hydroxide $(0.300 \mathrm{~g})$ in $\mathrm{methanol}(60 \mathrm{~mL})$ was heated at reflux for $16 \mathrm{~h}$. The reaction mixture was cooled to room temp. and concentrated in vacuo. Water (50 mL) and dichloromethane $(50 \mathrm{~mL})$ were then added and an aqueous solution of $\mathrm{HCl}(0.1 \mathrm{~N})$ was added until $\mathrm{pH}=3 \mathrm{was}$ achieved. The organic phase was then washed with water and dried with magnesium sulfate. After filtration and concentration in vacuo, imidazolidinone $9(0.368 \mathrm{~g}, 89 \%)$ was obtained as a thick white gum, which was used in the next step without further purification. - 9: IR (film): $\left.\bar{v}=3383 \mathrm{~cm}^{-1}, 3300,2949,1705,1634,1490,1452,1213 .-{ }^{1} \mathrm{H} \mathrm{NMR}(\mathrm{CDCl})_{3}\right): \delta=1.01(\mathrm{~d}, J$ $\left.=6.0 \mathrm{~Hz}, 3 \mathrm{H}, \mathrm{CH}_{3} \mathrm{CH}\right), 1.10-1.50\left(\mathrm{~m}, 6 \mathrm{H}, \mathrm{CH}_{2} \mathrm{CH}_{2} \mathrm{CH}_{2} \mathrm{CHNH}\right), 1.60\left(\mathrm{~m}, 2 \mathrm{H}, \mathrm{CH}_{2} \mathrm{CH}_{2} \mathrm{CO}\right), 2.29(\mathrm{t}, J=7.0 \mathrm{~Hz}, 2 \mathrm{H}$, $\left.\mathrm{CH}_{2} \mathrm{CO}\right), 3.52\left(\mathrm{qd}, J=7.9,6.0 \mathrm{~Hz}, 1 \mathrm{H}, \mathrm{CHCH}_{3}\right), 3.60\left[\mathrm{~m}, 1 \mathrm{H}, \mathrm{CH}(\mathrm{NH}) \mathrm{CH}_{2}\right], 3.90(\mathrm{~m}, 1 \mathrm{H}), 4.20(\mathrm{dd}, J=11.9,8.3 \mathrm{~Hz}, 1$ $\mathrm{H}), 4.39(\mathrm{~m}, 1 \mathrm{H}), 6.53$ (broad s, $\left.1 \mathrm{H}, \mathrm{HOCH}_{2}\right), 7.26-7.31$ (m, $\left.5 \mathrm{H}, \mathrm{CHPh}\right), 8.20$ (broad s, $\left.1 \mathrm{H}, \mathrm{HOCO}\right) .-{ }^{13} \mathrm{C} \mathrm{NMR}$ $\left(\mathrm{CDCl}_{3}\right): \delta=11.9\left(\mathrm{CH}_{3} \mathrm{CH}\right), 24.4,25.7,28.7,29.2\left(\mathrm{CH}_{2} \mathrm{CH}_{2} \mathrm{CH}_{2} \mathrm{CH}_{2} \mathrm{CH}_{2} \mathrm{CO}\right), 33.8\left(\mathrm{CH}_{2} \mathrm{CO}\right), 54.5,54.9(\mathrm{CHNH}, \mathrm{CH} 3 \mathrm{CH})$, $60.8(\mathrm{CHPh}), 64.2\left(\mathrm{CH}_{2} \mathrm{OH}\right), 127.0,127.6,128.5$ (Ar-C), 137.8 (ipso-Ar-C), $163.3(\mathrm{NCON}), 177.6(\mathrm{COOH})$.

Imidazolidinone 12: The same procedure described for the preparation of imidazolidinone $\mathbf{9}$ was applied to amine $\mathbf{1 1}(0.43$ $\mathrm{g}, \mathrm{mmol})$, and afforded imidazolidinone $12(0.368 \mathrm{~g}, 89 \%)$ as a white gum, which was used in the next step without further purification. - IR (film): $\bar{v}=3316 \mathrm{~cm}^{-1} 2930,1705,1450,1250,1074 .-{ }^{1} \mathrm{H} \mathrm{NMR}\left(\mathrm{CDCl}_{3}\right): \delta=1.14(\mathrm{~d}, J=5.6 \mathrm{~Hz}, 3 \mathrm{H}$, $\left.\mathrm{CH}_{3} \mathrm{CH}\right), 0.84-1.39\left(\mathrm{~m}, 6 \mathrm{H}, \mathrm{CH}_{2} \mathrm{CH}_{2} \mathrm{CH}_{2} \mathrm{CHNH}\right), 1.60\left(\mathrm{~m}, 2 \mathrm{H}, \mathrm{CH}_{2} \mathrm{CH}_{2} \mathrm{CO}\right), 2.29\left(\mathrm{t}, \mathrm{J}=6.6 \mathrm{~Hz}, 2 \mathrm{H}, \mathrm{CH}{ }_{2} \mathrm{CO}\right), 3.11(\mathrm{qd}$, $\left.J=5.9,5.6 \mathrm{~Hz}, 1 \mathrm{H}, \mathrm{CHCH}_{3}\right), 3.21\left[\mathrm{~m}, 1 \mathrm{H}, \mathrm{CH}(\mathrm{NH}) \mathrm{CH}_{2}\right], 3.95(\mathrm{~m}, 1 \mathrm{H}), 4.24(\mathrm{dd}, J=11.8,8.0 \mathrm{~Hz}, 1 \mathrm{H}), 4.39(\mathrm{~m}, 1 \mathrm{H})$, $6.51($ broad s, $1 \mathrm{H}, \mathrm{HOCH}), 7.22-7.34(\mathrm{~m}, 5 \mathrm{H}, \mathrm{CHPh}) .-{ }^{13} \mathrm{C} \mathrm{NMR}\left(\mathrm{CDCl}_{3}\right): \delta=18.1\left(\mathrm{CH} \mathrm{CH}_{3} \mathrm{CH}\right), 24.4,25.0,28.5,33.7$, $34.7\left(\mathrm{CH}_{2} \mathrm{CH}_{2} \mathrm{CH}_{2} \mathrm{CH}_{2} \mathrm{CH}_{2} \mathrm{CO}\right), 57.5,58.2\left(\mathrm{CHNH}, \mathrm{CH}_{3} \mathrm{CH}\right), 60.6(\mathrm{CHPh}), 64.2\left(\mathrm{CH}_{2} \mathrm{OH}\right), 127.1,127.6,128.5(\mathrm{Ar}-\mathrm{C}), 137.4$ (ipso-Ar- C), $162.7(\mathrm{NCON}), 177.5(\mathrm{COOH})$.

Methyl 6-[(4S,5R)-5-Methyl-2-oxoimidazolidin-4-yl]hexanoate (10): To a solution of imidazolidinone 9 (0.316 g, 0.946 $\mathrm{mmol})$ in methanol $(20 \mathrm{~mL})$ were added palladium(II) hydroxide on carbon $(20 \%, 0.500 \mathrm{~g})$ and two drops of concentrated hydrochloric acid. The suspension was stirred for $48 \mathrm{~h}$ at room temp. under hydrogen (1 atm). The suspension was filtered through a short pad of celite which was washed with methanol. The solution obtained was concentrated in vacuo. Chromatography on silica gel $\left(95: 5 \mathrm{MeOH} / \mathrm{CH}_{2} \mathrm{Cl}_{2}\right)$ afforded imidazolidinone $10(0.137 \mathrm{~g}, 64 \%)$ as a white solid.

10: $[\alpha]_{\mathrm{R}}^{26}=-2.3\left(c=0.865, \mathrm{CHCl}_{3}\right) .-\mathrm{IR}(\mathrm{film}): \bar{v}=3222 \mathrm{~cm}^{-1}, 2932,2861,1742,1699,1461,1171 .-{ }^{1} \mathrm{H} \mathrm{NMR}\left(\mathrm{CDCl}_{3}\right)$ : $\delta=1.12\left(\mathrm{~d}, J=6.2 \mathrm{~Hz}, 3 \mathrm{H}, \mathrm{CH}_{3} \mathrm{CH}\right), 1.24-1.37(\mathrm{~m}, 6 \mathrm{H}, \mathrm{CHCH} \mathrm{CH} \mathrm{CHNH}), 1.63(\mathrm{~m}, 2 \mathrm{H}, \mathrm{CH} \mathrm{CH} \mathrm{CO}), 2.30(\mathrm{t}, J=7.2$ $\left.\mathrm{Hz}, 2 \mathrm{H}, \mathrm{CH}_{2} \mathrm{CO}\right), 3.66\left(\mathrm{~s}, 3 \mathrm{H}, \mathrm{CH}_{3} \mathrm{O}\right), 3.60-3.70\left[\mathrm{~m}, 1 \mathrm{H}, \mathrm{CH}(\mathrm{NH}) \mathrm{CH}_{2}\right], 3.83\left(\mathrm{qd}, \mathrm{J}=7.7,6.2 \mathrm{~Hz}, 1 \mathrm{H}, \mathrm{CHCH}_{3}\right), 4.69$ (broad s, $1 \mathrm{H}, \mathrm{NH}), 4.95($ broad s, $1 \mathrm{H}, \mathrm{NH}) .-{ }^{13} \mathrm{C} \mathrm{NMR}\left(\mathrm{CDCl}_{3}\right): \delta=15.5\left(\mathrm{CH}_{3} \mathrm{CH}\right), 24.5,25.9,28.8,29.3 C \mathrm{H} \mathrm{CH} \mathrm{CH} \mathrm{CH}$ $\mathrm{CH} \mathrm{CO}), 33.7(\mathrm{CH} \mathrm{CO}), 51.2,51.3\left(\mathrm{CH} \mathrm{O}, \mathrm{CH}_{3} \mathrm{CH}\right), 55.8(\mathrm{CHNH}), 163.1(\mathrm{NCON}), 173.8\left(\mathrm{CO}_{2} \mathrm{CH}_{3}\right) .-\mathrm{C}_{11} \mathrm{H}_{20} \mathrm{~N}_{2} \mathrm{O}_{3}(228.3)$ : calcd. C 57.87, H 8.83, N 12.27; found C 58.02, H 8.59, N 11.95.

Methyl 6-[(4R,5S)-5-Methyl-2-oxo-imidazolidin-4-yl]hexanoate (ent- 10): Same characteristics as 10 except for the optical rotation. $[\alpha]_{\mathrm{D}}^{22}=+2.1\left(c=0.74, \mathrm{CHCl}_{3}\right)$. 
Methyl 6-[(4R,5R)-5-Methyl-2-oxo-imidazolidin-4-yl]hexanoate (13): The same procedure described for the preparation of com- pound 10 was employed. Starting from compound $12(0.316 \mathrm{~g}, 0.94 \mathrm{mmol})$, imidazolidinone $\mathbf{1 3}(0.137 \mathrm{~g}, 63 \%)$ was obtained.

13: $[\alpha]^{29}=+42.5\left(c=1.15, \mathrm{CHCl}_{3}\right) .-\mathrm{IR}(\mathrm{film}): \bar{v}=3239 \mathrm{~cm}^{-1}, 2942,2866,1741,1703,1440,1203 .-{ }^{1} \mathrm{H} \mathrm{NMR}\left(\mathrm{CDCl}_{3}\right):$ $\delta=1.10-1.70\left(\mathrm{~m}, 11 \mathrm{H}, \mathrm{CH}_{3} \mathrm{CH}, \mathrm{CH}_{2} \mathrm{CH}_{2} \mathrm{CH}_{2} \mathrm{CH}_{2} \mathrm{CHNH}\right), 2.30\left(\mathrm{t}, J=7.2 \mathrm{~Hz}, 2 \mathrm{H}, \mathrm{CH}_{2} \mathrm{CO}\right), 3.13\left[\mathrm{~m}, 1 \mathrm{H}, \mathrm{CH}\left(\mathrm{NH}^{2} \mathrm{CH}_{2}\right]\right.$, $3.33\left(\mathrm{~m}, 1 \mathrm{H}, \mathrm{CHCH}_{3}\right), 3.57\left(\mathrm{~s}, 3 \mathrm{H}, \mathrm{CH}_{3} \mathrm{O}\right), 5.85($ broad s, $1 \mathrm{H}, \mathrm{NH}), 6.00($ broad s, $1 \mathrm{H}, \mathrm{NH}) .{ }^{13} \mathrm{C} \mathrm{NMR}\left(\mathrm{CDCl}_{3}\right): \delta=21.0$ $\left(\mathrm{CH}_{3} \mathrm{CH}\right), 24.5,25.1,28.5,33.6,34.7\left(\mathrm{CH}_{2} \mathrm{CH}_{2} \mathrm{CH}_{2} \mathrm{CH}_{2} \mathrm{CH}\right.$ CO), 51.2 (CH O), 54.0 (CH CH), $60.2(\mathrm{CHNH}), 163.3(\mathrm{NCON})$, $173.8\left(\mathrm{CO}_{2} \mathrm{CH}_{3}\right) .-\mathrm{C}_{11} \mathrm{H}_{20} \mathrm{~N}_{2} \mathrm{O}_{3}(228.3)$ : calcd. C 57.87, $\mathrm{H}$ 8.83, N 12.27; found $\mathrm{C} 57.89, \mathrm{H} 8.65, \mathrm{~N} 12.05$.

Methyl 6-[(4S,5S)-5-Methyl-2-oxo-imidazolidin-4-yl]hexanoate (ent-13): Same characteristics as 13 except for the optical rotation. $[\alpha]_{\mathrm{D}}^{30}=-39.7\left(c=1.28, \mathrm{CHCl}_{3}\right)$.

\section{Acknowledgments}

This study was partially supported by the Bioavenir program financed by Rhône-Poulenc with the contribution of the Ministère de l'Education Nationale, de l'Enseignement Supérieur et de la Recherche. We thank Dr. Philippe Desbordes (Rhône-Poulenc Agro France) for helpful discussions.

\section{References}

${ }^{[1]}$ F. Escalettes, D. Florentin, B. Tse Sum Bui, D. Lesage, A. Marquet, J. Am. Chem. Soc. 1999, 121, 357123578, and refer-ences therein.

${ }^{[2]}$ F. Moreau, D. Florentin, A. Marquet, Tetrahedron 2000, 56, 2852293.

[3] A. Nudelman, A. Nudelman, D. Marcovici-Mizrahi, D. Flint, Bioorg. Chem. 1998, 26, 1572168.

${ }^{[4]}$ D. Lucet, L. Toupet, T. Le Gall, C. Mioskowski, J. Org. Chem. 1997, 62, 268222683.

${ }^{[5]}$ D. Lucet, S. Sabelle, O. Kostelitz, T. Le Gall, C. Mioskowski, Eur. J. Org. Chem. 1999, 258322591.

${ }^{[6]}$ E. Bosone, P. Farina, G. Guazzi, S. Innocenti, V. Marotta, U. Valcavi, Synthesis 1983, 9422944.

${ }^{[7]}$ V. du Vigneaud, D. B. Melville, K. Folkers, D. E. Wolf, R. Mozingo, J. C. Keresztesy, S. A. Harris, J. Biol. Chem. $1942,146,475$. 